\title{
Snail Fever of the Bladder in a Non-endemic Area
}

\author{
Zehra Kazmi, Muhammad Areeb Ashfaq, Daniya Umer, Romana Idrees and Muhammad Hammad Ather \\ Department of Surgery and Pathology, The Aga Khan University Hospital, Karachi, Pakistan
}

\begin{abstract}
Schistosomiasis is rarely seen in Pakistan and is generally not very high on the list of differential diagnoses. However, it is an important cause of haematuria in certain endemic areas. It can affect multiple organs including the bladder, liver and lungs. We present a case of a young Pakistani male, travelling to and from Africa, who presented with complains of haematuria and suprapubic discomfort. Final pathologic diagnosis was urinary schistosomiasis (hematobium species). This case is particularly notable for the characteristic sago nodules identified on cystoscopy. It is important to consider schistosomiasis in the differential of haematuria in such scenarios as it confers significant morbidity if left untreated.
\end{abstract}

Key Words: Schistosomiasis, Schistosoma hematobium, Haematuria, Urinary bladder.

How to cite this article: Kazmi Z, Ashfaq MA, Umer D, Idrees R, Ather MH. Snail Fever of the Bladder in a Non-endemic Area. J Coll Physicians Surg Pak 2020; 30(08):874-876.

\section{INTRODUCTION}

Urinary schistosomiasis is known to be endemic in 54 countries in Africa and the Middle East. ${ }^{1}$ While it is not endemic in Pakistan, the WHO listed the prevalence to be $<10 \%{ }^{2,3}$ The infection is caused when one comes in contact with cercariae, which are found in contaminated fresh water. These worms reside in the venous plexus of the bladder and the pelvis, where the female lays about 20 to 290 eggs/day. ${ }^{4}$ Once the schistosome eggs are deposited in the tissues, a granulomatous host response occurs. This infection has an incubation period of 10 to 12 weeks and hallmark features include hematuria and excretion of eggs in the urine. The most commonly affected organs are the seminal vesicles, distal ureters, and the urinary bladder. ${ }^{5}$

We report a case of urinary schistosomiasis in a young adult male who recently travelled to Africa. The histopathological and cystoscopic findings are discussed in detail, both prior to and post treatment with praziquantel.

\section{CASE REPORT}

A 24-year male with no comorbidity presented in urology clinic with the complaints of gross haematuria with clots for the past five months. Other lower urinary tract symptoms included burning, dysuria and bifid stream. There were no symptoms of constitutional or systemic compromise at that time. History of weight loss was absent.

Correspondence to: Dr. Zehra Kazmi, Department of Surgery and Pathology, The Aga Khan University Hospital, Stadium Road, Karachi, Pakistan

E-mail: zehra-kazmi@hotmail.com

Received: August 21, 2019; Revised: October 02, 2019; Accepted: October 09, 2019

DOI: https://doi.org/10.29271/jcpsp.2020.08.874

Social history was significant for smoking (5 pack years), occa- sional alcohol consumption (weekends) and high-risk sexual behaviour with multiple female partners. He is a frequent traveller to Europe, Africa and the Middle East and enjoyed fresh water swimming. He had recently returned from a trip to Africa, where he swam in the River Nile.

General physical, systemic and inguino-scrotal examination was unremarkable. Baseline investigations were all within normal limits. Complete blood count revealed eosinophilia (E: $20.8 \%$ ). Urine detailed report had 16 red blood cells/high power field (hpf). Urine Cytology was negative for malignancy. Plain CT scan of the kidneys and urinary bladder (CT KUB) showed soft tissue thickening in the left distal ureteric wall extending down and involving the left uretero-vesical junction and bladder trigone with periureteric fat infiltration and prominent lymph node in the left hemi-pelvis; there was mild left hydro-ureter and hydronephrosis (Figures 1 and 2 ).

On the basis of the above findings, after appropriate written and informed consent, we planned to proceed with cystoscopy and ureterorenoscopy.

Cystoscopy with bilateral retrograde pyelography, ureterorenoscopy, trans-urethral resection of bladder tumor and left double J (DJ) stent placement was performed seven days after initial presentation. Intra-operatively, the meatus, urethra and prostate were normal; right ureteric orifice was normal with a clear jet; the left ureteric orifice was completely obscured by papillary growths with calcific nodules. Nodular masses were visualized at the trigone. Multiple sessile growths were noted on the posterior wall (Figure 3). Bilateral retrograde pyelography demonstrated dilated middle and proximal ureters and the left distal ureter was kinked and appeared to have a tortuous course. $6 \mathrm{FrDJ}$ stent was inserted on the left side. Left ureteric biopsy and resection biopsies from the bladder growths were sent for analysis. Histopathology of the bladder specimens revealed exuberant chronic granulomatous inflammation with an 
organized granuloma, thecenter of which wasoccupied by aparasitic egg. Left ureteric biopsy also showed a parasitic egg with dense chronic inflammation. Photomicrography of the bladder showed Cystitis cystica glandularis (Figure 4) and granulomatous inflammation (Figure 5).

The Infectious Disease team was taken on board and the patient was started on tablet Praziquantel, $40 \mathrm{mg} / \mathrm{kg}$, orally thrice a day for one day only. As per their advice, a fecal occult blood test (FOBT) was sent which was positive and another dose of Praziquantel was repeated.

A follow up cystoscopy, approximately three months after the initial procedure, revealed an inflamed left ureteric orifice and inflammatory changes at the dome of the bladder. Theindwelling left DJ stent was removed. Histo-pathology of the bladder mucosa at this time revealed cystitis cystica and glandularis with no evidence of schistosomiasis. At present, the patient is doing well and does not have any lower urinary tract symptoms

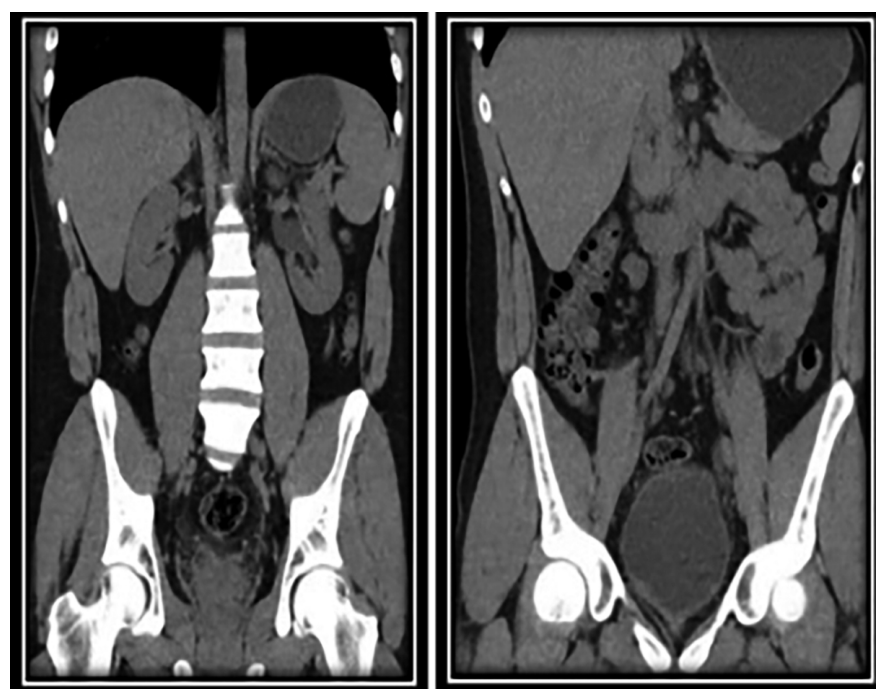

Figure 1: Coronal view of CT KUB showing left hydronephrosis and diffuse thickening of the bladderwall.
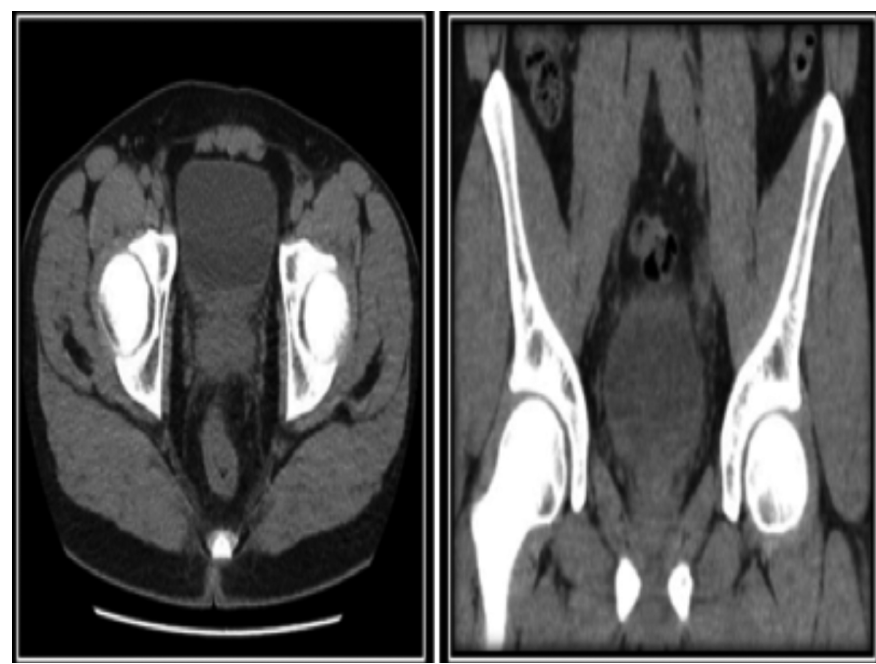

Figure 2: Axial images of plain CT KUB demonstrating soft tissue thickening in the distal left distal ureteric segment involving the left ureterovesical junction, and diffuse bladder wall thickening

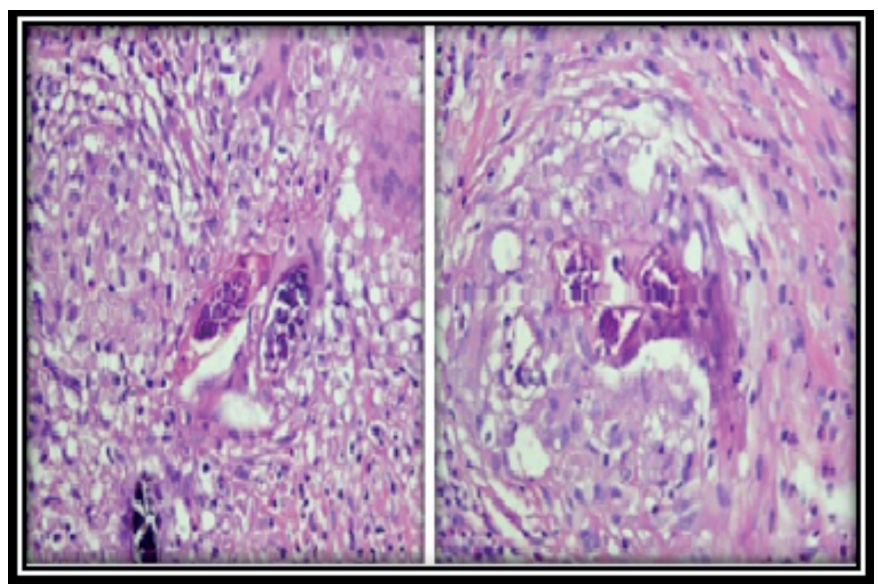

Figure 3: Intra-operative cystoscopic findings demonstrating sessile bladder growths mostly at the trigone, with prominent calcific nodules.

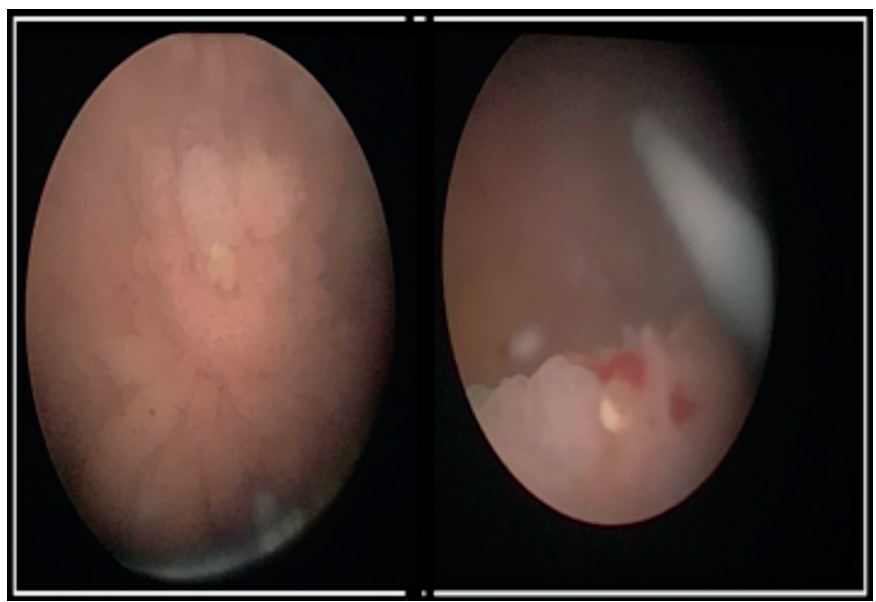

Figure 4: Photomicrography of urinary bladder showing Schistosoma hematobium egg nested at the center of an organized granuloma.

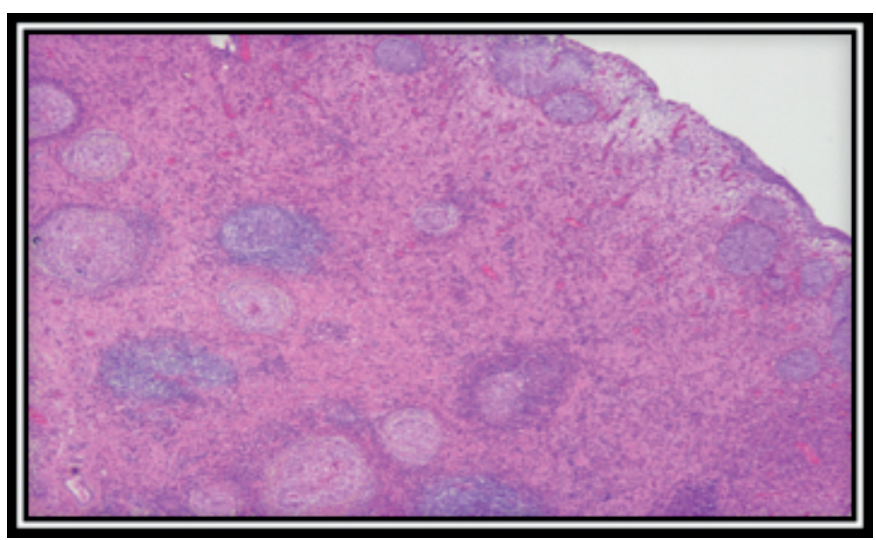

Figure 5: Photomicrography of urinary bladder showing chronic granulomatous inflammation.

\section{DISCUSSION}

Currently, over 200 million people in 74 countries worldwide are infected with schistosomiasis, especially in sub-Saharan Africa. This infection occurs by trematodes belonging to the schistosoma genus. Based on the geographical area, the main disease causing species are schistosoma mansoni (Africa and South America), S. haematobium (Africa and the Middle East), and S. Japonicum (East Asia). In addition to being classified as one of 
the neglected tropical diseases by WHO, this tropical parasitic infection is also considered to be one with the greatest socio-economic impacts. ${ }^{4}$

S. haematobium is the cause of bladder schistosomiasis. The fresh water snails are the intermediate hosts, and they release the larvae (cercariae) into water. Once they have penetrated into the human skin, they travel to the liver and lungs. 1-2 days after penetration, a maculopapular rash, known as Swimmer's itch, results. Katayama fever occurs in the following few weeks with presentation of weight loss, cough, malaise, and urticarial rash. ${ }^{5}$ As the larvae mature into adult worms, they travel towards the pelvic veins and begin depositing eggs along the bladder wall vessels. These eggs are then excreted out in urine, which in turn, results in granuloma formation with chronic inflammatory response. In rare cases, it can cause metaplasia of the bladder epithelium which results in squamous cell carcinoma. ${ }^{6,7}$

Most infections caused by this tropical parasite occur from the age of about 4 years to a peak age of 15-20 years. Since non-specific symptoms such as suprapubic pain, dysuria, and terminal hematuria occur in urinary schistosomiasis, a CT scan is considered to be better in diagnostic terms compared to ultrasound. A CT scan gives specific information about the affected organs, degree of calcification, and the extent of lesions.

The most accurate tool for the evaluation of response to treatment is cystoscopy with histological examination of biopsy material.

Late complications of bladder schistosomiasis include progression to calcification and fibrosis with the development of squamous metaplasia. In rare instances, it may progress to squamous cell carcinoma. Ureters may show fibrosis, calcification and irregular dilatations due to stricture formation. Renal involvement may also be seen in late stages resulting in hydronephrosis, renal calculi and pyonephrosis. ${ }^{5,8}$

Praziquantel is the established anthelmintic treatment drug used to destroy the adult worms. $80-90 \%$ of schistosomiasis patients are cured with this treatment regimen. In those not cured, it causes a $90 \%$ reduction in urinary egg excretion., ${ }^{9,10}$

The chronic fibrotic changes in the bladder wall cannot be cured. The patient should be followed up for the next 6 months for confirmed eradication of eggs. Currently, an irradiated cercariae vaccine is under clinical trial. ${ }^{11}$

Schistosomiasis should be on the list of differential diagnoses in patients presenting with hematuria, and those returning to Pakistan from snail fever endemic areas. Due to non-specific symptoms, the disease is difficult to diagnose, and often the diagnosis is made, when it is in an advanced stage. Bladder symptoms can easily be missed in non-endemic areas. A thor- ough history and awareness of the disease can help avoid any delay in treatment.

\section{CONFLICT OF INTEREST:}

The authors declare no conflict of interest.

\section{PATIENT'S CONSENT:}

Informed consent was obtained from the patient to publish the data concerning this case.

\section{AUTHORS' CONTRIBUTION:}

ZK: Manuscript writing, drafting and final submission; accountable for all related queries.

MAAs: Manuscript drafting and literature search.

DU: Manuscript writing and literature search.

RI: Histopathologic slides and details.

$\mathrm{MH}$ : Supervised manuscript writing, reference selection and finalsubmission.

\section{REFERENCES}

1. Chitsulo L, Engels D, Montresor A, Savioli L. The global status of schistosomiasis and its control. Acta Tropica 2000; 77(1):41-51.

2. Rollinson D, Knopp S, Levitz S, Stothard JR, Tchuenté L-AT, Garba A, et al. Time to set the agenda for schistosomiasis elimination. Acta Tropica 2013; 128(2):423-40.

3. Schistosomiasis W. Number of people treated worldwide in 2009. Wkly Epidemiol Rec 2011; 86:73-80.

4. Inobaya MT, Olveda RM, Chau TN, Olveda DU, Ross AG. Prevention and control of schistosomiasis: a current perspective. Res Rep Trop Med 2014; 2014(5):65-75.

5. Ghoneim M. Bilharziasis of the genitourinary tract. BJU Int 2002; 89(Suppl 1):22-30.

6. Roure S, Valerio L, Pérez-Quílez O, Fernández-Rivas G, Martínez-Cuevas O, Alcántara-Román A, et al. Epidemiological, clinical, diagnostic and economic features of an immigrant population of chronic schistosomiasis sufferers with long-term residence in a non-endemic country (North Metropolitan area of Barcelona, 2002-2016). PLoS One 2017; 12(9):e0185245.

7. Conlon C. Schistosomiasis. Medicine 2005; 33:64-7.

8. Neal PM. Schistosomiasis-An Unusual Cause of Ureteral Obstruction A Case History and Perspective. Clin Med Res 2004; 2(4):216-27.

9. Picquet M, Vercruysse J, Shaw DJ, Diop M, Ly A. Efficacy of praziquantel against Schistosoma mansoni in northern Senegal. Trans R Soc Trop Med Hyg 1998; 92(1):90-3.

10. Reich MR, Govindaraj R, Dumbaugh K, Yang B-m, Brinkmann A, El-Saharty S, et al. International strategies for tropical disease treatments: Experiences with praziquantel. Geneva: World Health Organization; 1998.

11. Siddiqui AA, Siddiqui BA, Ganley-Leal L. Schistosomiasis vaccines. Hum Vaccin 2011; 7(11):1192-7. 